\title{
Mitteilungen.
}

\section{Zum hundertsten Geburtstag von J. K. Qvigstad.}

Ein Jubiläum seltener Art feierte man in der wissenschaftlichen Welt am 4. April 1953, als Staatsrat Rektor Just Knud Qvigstad hundert Jahre alt wurde. Wenn ein Menschenleben frei von siechtum diese äusserste Grenzzone erreicht, ist das stets ein Ereignis, das uns erfreut und anrührt, aber am grössten ist die Festfreude, wenn wir feststellen können, dass dieses aussergewöhnlich lange Leben ein Geschenk des Scchicksals gewesen ist, die unbedingt erforderliche Voraussetzung, um ein grosses Lebenswerk zu Ende zu führen. Es entspricht ja der Ordnung der Natur, dass ein Hundertjähriger ein oder zwei Jahrzehnte vollständiger, verdienter Ruhe hinter sich hat, aber in dieser Hinsicht ist Qrigstad eine grossartige Ausnahme: Noch bei sinkender Somne hat er unermüdlich und erfolgreich die emsige Arbeit beim Schein seiner Studierlampe fortgesetzt, die aus der abgelegenen stadt Tromso weithin geleuchtet hat. In den nordischen Ländern, wo die Erforschung der Lappen und der lappischen sprache mit ihren engen Beziehungen zu manchen anderen Forschungsgebieten ihren Platz im Kreise der nationalen Wissenschaften hat, anerkennt eine zahlreiche Schar ron Forschern Qvigstad als ihr Vorbild, dessen Persönlichkeit eine sammelnde und rerbindende Kraft bedeutet; und auch überall, wo es sonst noch Vertreter der finnisch-ugrischen Sprachwissenschaft und Volkskunde gibt, nennen diese seinen Tamen mit Empfindungen aufrichtiger Verehrung und Dankbarkeit.

Qvigstad, der als Sohn des Kreisarztes von Lyngen in Finnmarken geboren wurde, kam mit zehn Jahren in die "Lateinschule" in Tromso und bestand im Jahr 1869 als erst 16jähriger die Reifeprüfung mit dem bestmöglichen Zeugnis. Ebenso ausgezeichnete Fortschritte machte der frülreife Junge in der Universität, wo er nach dem Studium der Philosophie und Sprachwissenschaft das philosophische Kandidatenexamen im Jahr 1874 ablegte. Schon im folgenden Jahre kehrte er nach Tromso zurück, um dort zuerst Lehrer an der Oberschule und seit $1878 \mathrm{am}$ seminar zu werden. Im Iienste dieses Seminars verbrachte Qvigstad über 40 Jahre; seit 1883 wirkte er als Leiter der Lehranstalt, ein Amt, das 1902 mit dem Titel eines Rektors ausgezeichnet wurde.

Qrigstad war bereits seit jungen Jahren mit den Lappen 
bekannt, aber die eigentliche Veranlassung dazu, dass er in diesem Bereich seine Lebensaufgabe fand, waren die von ihm übernommenen Lehrverpflichtungen. Im Tromsoer Seminar wurden die zukünftigen Volkserzieher auch in der lappischen Sprache unterrichtet und Qvigstad erhielt zufällig gerade die Lehrerstelle, zu deren Anfgabengebiet dieser Enterricht gehörte. Cm sich mit dieser Aufgabe vertraut zu machen, unternahm er im IIärz 1878, also vor genau 75 Jahren, eine Studienreise zu den Lappen ron Kautokeino. Dieser Reise sind dann viele andere gefolgt und als Ergebnis derselben hat sich eine Materialkollektion angesammelt, die nicht ihresgleichen findet.

Die Resultate dieser Studien zeigten sich bald: schon 1881 war Qrigstad so weit, dass er in der Öffentlichkeit als mit der lappischen Sprache gut vertrauter Forscher auftreten konnte. Sein wissenschaftliches Erstlingswerk, die in den Teröfentlichungen der Finnischen Gesellschaft der Wissenschaften erschienenen "Beiträge zur Vergleichung des rerwandten Wortvorrathes der lappischen und der finnischen Sprache» (128 S. 4:o), ist keine blosse etymologische Untersuchung, sondern ein Werk, das wesentlich mehr bringt, als sein Titel verspricht. Im einleitenden Teil wird ein sehr ins Einzelne gehendes phonetisches und morphologisches Tergleichsmaterial dargeboten, in dem die Verhältnisse des Lappischen und des Fimnischen nebeneinandergestellt sind. Das etymologische Wörterverzeichnis, das den Charakter eines vollständigen Inventars hat, umfasst (auf die Stammwörter reduziert) etwa 900 lappisch-finnische Wortvergleichungen. I)iese erstaunlich grosse Zahl erklärt sich dadurch, dass sich darunter viel späte finnische Lehnwörter finden - worüber sich der Verfasser selbst durchaus im Klaren war - sowie auch solche Zusammenstellungen, die man heute nicht mehr für richtig halten kann. In Anhängen werden die wichtigsten skandinavischen Lehnwörter der lappischen Sprache sowie das dem Verfasser bekannte Wortgut finnischugrischer Herkunft, das Entsprechungen im Jordwinischen und Tscheremissischen, aber nicht im Finnischen hat, aufgezählt. In dem letztgenannten Anhang finden wir etwa 20 richtige Zusammenstellungen, die Qrigstad selbst gemacht hat.

$\dot{L}^{*}$ ber die "Beitläge" ist zu sagen, dass sie die erste vergleichende Untersuchung sind, die die Geschichte der lappischen Sprache vielseitig beleuchtet. I)araus wird auch die Insicht des Terfassers uber die Stellung dieser Sprache in der finnischugrischen Sprachfamilie deutlich ersichtlich: Als Vergleichs- 
objekte sind in erster Linie das Finnische und in zweiter Linie die wolgafinnischen Sprachen gewähIt. Die Auffassung, dass das Lappische diesen Gruppen am nächsten stehe, dürfte in den 80er Jahren des 19. Jahrhunderts noch allgemein verbreitet gewesen sein. So sagt August Ahlqvist, um nur ein Beispiel zu nennen, in der deutschen Ausgabe seines im Quellenverzeichnis der Qvigstadschen Untersuchung erwähnten Werkes "De restfinska språkens kulturord" ("Die Kulturwörter der westfinnischen Sprachen", 1875), dass das Lappische ein Verbindungsglied zwischen den "baltisch-finnischen" und den "ostfinnischen" Sprachen bilde: von den letzteren scheine das Mordwinische mit der lappischen Sprache am nächsten verwandt zu sein. Ohne Bedenken kann man sagen, dass diese Ansicht wesentlich besser den Tatsachen entspricht als beispielsweise die bekannten Theorien ron Budenz und Setälä, ron denen der erste das Lappische mit den ugrischen und permischen Sprachen in Verbindung brachte, während der zweite es als eine alleinstehende Abzweigung ron der finnisch-ugrischen Ursprache betrachtete. Eine Besonderheit der Darstellungsweise von Qvigstad, die auch in seiner ganzen späteren Produktion erhalten bleibt, ist die ausserordentliche Knappheit des Ausdrucks; der Forscher lässt lieber das gut geordnete Material sprechen, als dass er selbst dessen Einzelheiten analysiert. Für die späteren Forscher ist diese Grosszügigkeit vorteilhaft gewesen: Man kann einige Fälle aufzeigen, in denen wichtige sprachgeschichtliche Einsichten sich mit Sicherheit auf der Grundlage des IIaterials der »Beiträge» herauskristallisiert haben.

In den nächsten Jahren nahm Qrigstad die Gewohnheit an, jeden Sommer Reisen zur Erforschung der lappischen Dialekte zu unternehmen. Indem er bei den Fjell-, See-, Lule-, Pite- und Südlappen umherwanderte, lernte er die schwer festzulegenden Dialektgrenzen besser kennen als irgendein anderer vor ihm oder nach ihm. Beim sammeln der Wörter konzentrierte sich Qvigstad auf bestimmte Sondergebiete, die ihn als Forscher interessierten. Yor allem hatte er beschlossen, an die Klärung der Geschichte des reichhaltigen skandinarischen Lehnwortgutes im Lappischen zu gehen. Sein Vorbild war Vilhelm Thomsens hervorragendes Werk "Den gotiske sprogklasses indflydelse paa den finske". Darin waren riele Elemente germanischer Herkunft auch in der lappischen Sprache behandelt worden, von denen Thomsen einige schon etwa ins 3. Jahrhundert n. Chr. datierte. Qvigstads Meinung nach waren Thomsens Kriterien nicht bindend; er sah keine Veranlassung, auch die ältesten Lehn- 
wörter für früher zu halten als die Wikingerzeit. Diese seine Auffassung brachte er im Jahr 1893 in seinem 365̃seitigen Hauptwerk "Nordische Lehnwörter im Lappischen" zum Ausdruck, das in seiner Anlage mit den "Beiträgen" nah verwandt ist. Dessen unvergänglicher Wert liegt in dem mit ziemlich erschöpfender Genauigkeit gesammelten, wenigstens 2.500 Wörter umfassenden Material; zur Feststellung der Verbreitung und der Lautverhältnisse der Wörter hat der Verfasser dutzendweise .Iundarten erforscht. Ein recht grosser Teil dieses Wortschatzes stammt zweifellos aus dem ditnorwegischen oder auch aus neunorwegischen Dialekten, worauf Qvigstad hingewiesen hat, aber anderseits finden sich nach dem heutigen Stande der Wissenschaft im Lappischen auch erheblich ältere Entlehnungen, die bereits aus dem Urnordischen übernommen worden sind. K. B. Wiklund zeigte im Jahr 1892, also ungefähr zur selben Zeit, als Qrigstads Werk, das rier Jahre auf den Druck warten musste, erschien, wie namentlich das spärliche skandinavische Wortgut in den peripheren kolalappischen Iundarten deutlich für diese Auffassung spricht. Jetzt allerdings vermissen wir schon sehr eine gründliche, moderne Klarlegung der Chronologie und Lautgeschichte der skandinavischen Lehnwörter des Lappischen. Werden sich in unseren westlichen Nachbarländern noch Forscher finden, die an dieses anspruchsvolle Thema herangehen, dessen Erforschung sicherlich auch der Erforschung der nordischen Sprachen nützen würde? In Qrigstads Werk läge das Material fertig ror!

Qvigstads dritte grundlegende sprachwissenschaftliche Cntersuchung, die im Jahr 1925 erschienene Schrift "Die lappischen Dialekte in Norwegen" (20 s. 4:0), stammt aus seiner ungeheuer produktiven spätzeit, die 1920 begann, als er das Rektorat des Seminars niedergelegt hatte und sich, gesichert durch eine ihm bewilligte Ehrenpension, ausschliesslich wissenschaftlicher Arbeit widmete. Seine Einteilung des Norwegischlappischen und der diesem benachbarten Dialekte in Cntermundarten bedeutet einen grossen Fortschritt gegenüber den früheren Abgrenzungen. Sie ist eine Synthese jahrzehntelanger Forschungen, in der der Verfasser seinen Standpunkt kurz, aber überzeugend mit phonetischen und manchmal auch morphologischen Argumenten begründet. $\mathrm{Zu}$ den interessantesten Zügen dieser Synthese gehört die Charakteristik und Kartographierung der seelappischen Mundarten, die Qvigstad persönlich wohl am nächsten stehen.

Als bester Kenner der lappischen Literatur hat Qrigstad in dem Material der alten lappischen Schriftsprache, das 
einen achtenswerten Umfang besitzt, viele für die Sprachwissenschaft wichtige Entdeckungen gemacht. Seine Monographien "Die Sprache in Tornæus' Manuale Lapponicum" (1933; $13 \mathrm{~S}$.$) und "Sproget i Graans Manuale Lapponicum"$ (1947; $22 \mathrm{~S}$.$) , deren Gegenstand zwei in den Jahren 1648$ und 1669 erschienene Monumentalwerke sind, bieten eine Lektüre von grösstem Reiz. Die vom Verfasser behandelten Besonderheiten und Archaismen aus den Gebieten der Laut- und Flexionslehre, der Wortklassen, des Wortschatzes und der Syntax bringen Licht in manche Fragen und regen zum Aufwerfen neuer Fragen an.

So viel auch Qvigstad während seiner Wissenschaftlerlaufbahn lautgeschichtliche Dinge hat behandeln müssen, so wenig Sonderuntersuchungen aus diesem bei den Forschern im allgemeinen beliebten Gebiet hat er verfasst. $Z u$ erwähnen sind hier ror allem zwei Aufsätze "Dobbeltkonsonant i forlyd i lappisk" $(1945 ; 20 \mathrm{~S}$.$) und "Das anlautende h$ im Lappischen" (1946; $15 \mathrm{~S}$.$) , in denen der über 90jährige Forscher ein wirk-$ lich wertvolles und gut kommentiertes Material vorlegt.

Seben der Erforschung der skandinavischen Lehuwörter waren Qvigstads Lieblingsobjekt auf seinen Sammelreisen die lappischen Ortsnamen. Im Lauf der Jahre häuften sich gewaltige Mengen davon in seinen Aufzeichnungsbüchern. Es ist ganz natürlich, dass in jener Umgebung, die Qvigstads wichtigstes Arbeitsfeld bilden sollte, nämlich an der von drei Nationalitäten besiedelten Eismeerküste, die Aufmerksamkeit des Erforschers siedlungsgeschichtlicher Fragen sich stark auf die Ortsnamen richtet. Seine Ortsnamensammlungen veröffentlichte Qrigstad in zwei umfangreichen Untersuchungen "De lappiske stedsnavn i Troms fylke» (1935; 162 S.) und "De lappiske stedsnarn i Finnmark og Nordland fylker" $(1938 ; 275 \mathrm{~S}$.$) . In der ersten sind etwa 6.000$ und in der zweiten etwa 10.000 lappische Ortsnamen aufgezählt, die systematisch mit Hilfe der Karte gesammelt worden sind. Die Einleitung des Werkes über die Ortsnamen des Amtes Tromsø ist eine Quelle von erstrangigem Wert für die Erforschung der Siedlungsgeschichte. Dieselben Fragen hat Qvigstad schon früher in der Einleitung seines Buches "Nordische Lehnwörter" und in seiner Spezialuntersuchung " schen Ortsnamen im Amt Tromso" (1913; 7 S.) erörtert, wobei er u. a. die Auffassung vertrat, dass die norwegische Besiedlung an den Segelschiffahrtswegen entlang der Küste älter sei als die lappische. Beide Schriften enthalten auch eine beträchtliche Jenge finnischer Ortsnamen. Die Chronologie dieser Ortsnamen und die Einwanderung der finnischen Neu- 
siedler setzt Qvigstads Untersuchung "Den kranske indrandring til Nord-Norge» $(1921 ; 90 \mathrm{~S}$.$) ausführlich ausein-$ ander. Auf Grund genauer Quellenforschungen zeigt der Terfasser, dass die Einwanderungsbewegung im 18. Jahrhundert und zwar während des Nordischen Krieges eingesetzt hat. Ausser der Siedlungsgeschichte bieten die lappischen Namen in Qvigstads Ortsnamenteröffentlichungen z. B. der Erforschung des Volksglaubens ein gutes Material; hier und da tauchen solche Namen auf wie ailigâs und bâsse 'heilig', aldâ 'Opferstelle', gânišs 'Kobold', gufittâr und uldâ 'ein Unterirdischer', halde 'Schutzgeist', sieide 'Opferstein' usw. Am Ende des zuletzt erwähnten Buches findet sich ein wertroller Überblick über die allgemeine Struktur der lappischen Ortsnamen. Eine gute Ergänzung hierzu bildet die Einleitung ron Qrigstads dritter Cntersuchung über die Ortsnamen "De lappiske appellative stedsnavn》 (1944; 82 S.). Die Schrift selbst gehört nicht ins Gebiet der eigentlichen Ortsnamenforschung, sonderu ist eher ein topographisches Wörterverzeichnis der lappischen Dialekte oder man könnte auch sagen - der lappischen Sprachen, dessen 945 Wortartikel u. a. gute etymologische Erklärungen enthalten.

Die übrigen Namenpublikationen Qvigstads, die im wesentlichen den Charakter geordueter Materialsammlungen liaben, umfassen die lappischen Bezeichnungen für Pflanzen (1901; 24 S.), Vögel (1902; $25 \mathrm{~S}$.$) , sonstige Tiere (1904 ; 48 \mathrm{~S}$.$) und$ Sterne $(1921 ; 10 \mathrm{~S}$.$) .$

Mit der Veröffentlichung lappischer Texte begann Qvigstad in den Jahren 1887/88, als zwei Sammlungen lappischer Märchen und Erzählungen erschienen, die er und Pfarrer G. Sandberg gesammelt hatten. Die folgenden, nach einer langen Pause herausgekommenen Textveröffentlichungen "Lappischer Aberglaube" (1920; 95 S. 4:0) und "Lappische Sprichwörter und Rätsel» (1922; 114 S. 4:0) verfolgen einen doppelten Zweck: Sie sind sowohl Sprachbeispiele als auch mit vielen sachkundigen, vergleichenden Literaturhinweisen versehene Materialsammlungen für die Erforscher der Mythologie und Folklore. Den Grund zur Erforschung dieser Volksüberlieferung hat Qvigstad in den Vorworten der beiden Veröffentlichungen selbst gelegt, wo er feststellt, dass sie in der Hauptsache nicht bodenständig, sondern von den Nachbarn, den Norwegern und Finnen, übernommen worden ist. Die auch heute noch gewöhnliche Auffassung, dass unter den Lappen uralte Zauberbräuche blühen, wird also auch hier als Einbildung entlarvt. Die Aufzeichnungen aus dem Gebiet 
des Aberglaubens, 387 an Zahl, sind grösstenteils die Ausbeute eingeborener lappischer Sammler. Cnter diesen Sammlern befinden sich u. a. etwa zehn Tolksschullehrer, vermutlich zum grössten Teil Qvigstads frühere Schüler aus dem Tromsoer Seminar, die ron ihm die Anregung zur Sammeltätigkeit erhalten haben. Die Zahl der Sprichwörter beträgt 760, die der Rätsel 132. Auch in diese Veröffentlichung hat Qrigstad Aufzeichnungen anderer Sammler mit aufgenommen. Die Sprachbeispiele, die er bei den Südlappen in den 80er Jahren des 19. Jahrhunderts gesammelt hatte und die wert rolle, rerschwindende Folklore sind, veröffentlichte Qvigstad im Jahr 1924 unter dem Titel "Lappische Erzählungen aus Hatfjelldalen" (63 S. 4:0). Die im nächsten Jahr erschienene Sammlung "Lappische Texte aus Kalfjord und ILelgös" (20 S. 4:0) ist sprachlich äusserst merkwürdig. Im Sommer 1886 erfuhr Qrigstad zufällig, dass auf ein paar Inseln in der Nähe ron Tromso einige Lappen wohnten, die ihren eigenen, ron der Sprache der Lmgebung abweiohenden Dialekt sprachen. Er reiste hin und konnte feststellen, dass er einen rorher nicht bekannten Dialekttypus, die südlichste seelappische IIundart, entdeckt hatte, die damals schon im Aussterben begriffen war und nur noch ron 17 betagten Personen gesprochen wurde. Obwohl dieser Dialekt num schon seit Jahrzehnten tot ist, steht er dank Qvigstad nach wie vor der Forschung zur Verfügung. Die »Lappische Wetterkunde» (1932; 47 S. 4:0) enthält etwa 400 Wetter vorzeichen. Das norwegischlappische Material dieser Wetterkunde hat der Herausgeber selbst gesammelt. Aus den übrigen lappischen Gebieten hat er Vergleichsmaterial aufgenommen, das jedoch nur in deutscher Übersetzung veröffentlicht ist.

In den Jahren 1927-29 veröffentlichte Qrigstad seine wichtigste Tertsammlung, das Honument der norwegischlappischen Folklore "Lappiske eventyr og sagn", dessen vier umfangreiche Teile insgesamt über 2.300 Seiten umfassen. Den Inhalt bilden etwa 1.000 Märchen und Erzählungen mit norwegischen Übersetzungen. Reichlich die Hälfte des ganzen Materials ist rom Herausgeber selbst gesammelt. Von seinen Helfern sind die verdientesten der aus Inari gebürtige Johan Aikio und der auch als Reichstagsmitglied bekannte Isak Saba, beide aus Nesseby. Mit Hilfe dieses grossen Werkes gewinnen wir einen guten Einblick in Art und Quantität der lappischen Folklore. Die Skala erstreckt sich ron den durch besonders echte lokale Färbung ausgezeichneten Tier märchen, Stallosagen, Metamorphosensagen, die Menschen inTiergestalt darstellen, Kriegsüberlieferungen und Erzählun- 
gen von Zauberern, Unterirdischen, Seegespenstern sowie sonstigen merkwürdigen Wesen bis zu jungen internationalen Wandermärchen und -erzählungen. Am Ende jedes Teils finden sich zahlreiche Anmerkungen des Herausgebers. Diese werden ergänzt durch die Abhandlung "Lappische Märchenund Sagenvarianten» (1925; 64 S.), die Qrigstad nach den bekannten Typenverzeichnissen ron Antti Aarne verfasste und in der Reihe "FF Communications" reröffentlichte. Qvigstad hat sogar eine kleine Kollektion von ihm selbst gesammelter Erzählungen der Finnen aus Finnmarken herausgegeben ("Finske fortallinger fra Kranangen og Nord-Reisa", $1925 ; 31$ S.).

Auch als Erforscher des Volksglaubens und der Volkskunde hat sich Qvigstad betätigt. Die Schrift "Lappische Opfersteine und heilige Berge in Norwegen" (1926; 40 S. 4:0) beginnt mit einem Überblick über die Literatur, die die Sieide-Verehrung und die heiligen Stätten behandelt, worauf eine Aufzählung sämtlicher Götzendienststätten folgt, die der Verfasser entweder auf Grund der im Volk lebendig gebliebenen Tradition oder aus schriftlichen Quellen kennt. Qvigstads wichtigstes Werk über Volkskultur trägt den Titel "Lappische Heilkunde mit Beiträgen von K. B. Wiklund» (1932; 270 S.). Diese Untersuchung über die volkstümliche Heilkunde der Lappen ist grösstenteils deskriptiv; am Ende finden sich 20 Seiten Hinweise auf die entsprechenden Verfahren anderer Völker und sonstige Nachbemerkungen. Die Gründlichkeit der Untersuchung wird durch das Verzeichnis der gedruckten und ungedruckten Quellen bewiesen, das sechs Seiten Petitdruck umfasst. Dem Thema nach hängt mit diesem Werk eng zusammen eine Abhandlung über das für die Lappen sehr wichtige Gebiet der Tierkrankheiten unter der ¿̈berschrift "Den tamme rens sykdommer" (1941; $56 \mathrm{~S}$.).

In reinen Materialsammlungen, die mit Forschungen wie den soeben erwähnten zusammenhängen, sei zunächst als wichtigste eine Kollektion alter Handschriften genannt, die Aufschluss über den volkstümlichen Glauben der Lappen geben, "Kildeskrifter til den lappiske Mythologi»; den ersten Teil derselben (90 S.) gab Qvigstad im Jahr 1903 heraus, während der zweite Teil, den der Missionsprediger Isak Olsen in der ersten Hälfte des 18. Jahrhunderts unter dem Titel "Om lappernes vildfarelser og overtro» verfasst hatte und der rom Herausgeber wiederentdeckt worden war, im Lmfang von 101 Seiten im Jahr 1910 erschien. Ergänzungen zu dieser Sammlung enthält die Veröffentlichung "Nordlands og Troms finner i eldre hândskrifter” (1943; $27 \mathrm{~s}$.$) . Den Erforschern$ 
der Renntierzucht und der umherziehenden Lebensweise der nomadisierenden Lappen bieten die con Qvigstad zusammen mit Wiklund in zwei Teilen reröffentlichten "Dokumenter angaaende flytlapperne" (1909; 538 - 524 S.) ein gutes Material. Im Jahr 1950 erschien die 65seitige Darstellung des Lebens der Seelappen "Om sjøsamene». Diese einfache, aber unterhaltende und nützliche Schilderung hat Qrigstad aus dem lappischen Manuskript eines seiner ehemaligen Schüler, des Lehrers Anders Larsen ins Norwegische übersetzt und veröffentlicht. Seiner Gewohnheit nach hat er zahlreiche ergänzende Hinweise am Ende der Beschreibung hinzugefügt. In diesen ist auch die allerneueste Literatur genau berücksichtigt, was ron der ungebrochenen geistigen Kraft des Forschers zeugt.

Qvigstad hat sich mit dem lappischen Schrifttum nicht nur in seiner Eigenschaft als Forscher beschäftigt, sondern es auch als seine Pflicht angesehen, aktir an diesem Schrifttum mitzuarbeiten. Auch auf diesem Gebiet hat er Leistungen vollbracht, die das gewöhnliche Mass übersteigen, so dass er neben Pastor N. V. Stockfleth und Professor J. A. Friis zu den grossen Namen der norwegischlappischen Literatur gehört. Ihm wurde die ehrenvolle Aufgabe übertragen, der lappischen Bibel die endgültige sprachliche Form zu geben. Das Neue Testament war in der Utbersetzung ron Stockfleth schon 1840 erschienen und die rorläufige $\dot{\mathrm{C}}^{*}$ bersetzung des Alten Testaments besorgte der Lappe Lars Hetta aus Kautokeino, Qvigstads erster Gewährsmann. Im Jahr 1885 wurden Friis und Qvigstad gemeinsam mit der Redaktion der Bibelübersetzung beauftragt, aber nachdem 1891 Friis erkrankt war, lag die Verantwortung für die ganze Arbeit auf Qvigstad. Als Ergebnis zehnjähriger opferbereiter und mühevoller Tätigkeit, in deren Verlauf der gelehrte und gewissenhafte Herausgeber sein Manuskript ständig mit dem hebräischen und dem griechischen Urtext verglich, erschien die Bibel im Jahr 1895, un von da an vom gläubigen lappischen Kirchenvolk fleissig gelesen zu werden. Insgesamt etwa 50 lappische Druckerzeugnisse hat Qrigstad übersetzt oder sprachlich kontrolliert. Im Jahr 1899 veröffentlichten er und Wiklund zusammen das wichtige Nachschlagewerk "Bibliographie der lappischen Litteratur» (MSFFOu XIII; 163 S.).

Unser Überblick über Rektor Qrigstads Lebensarbeit würde unvollständig bleiben, wenn wir seine Leistungen auf dem Gebiet der Heimatforschung übergehen würden. Mit diesem Namen können wir die Seite ron Qvigstads Tätigkeit bezeichnen, die in sachbedingt allgemeinverständlichen 
Formen vor allem die Verbindung zu seinen eigenen Landsleuten und unter ihnen besonders zu den Einwohnern seiner Heimatprovinz suchte. Die Stadt Tromso und die sie umgebende Landschaft sind durch Qvigstads ganzes Leben hindurch Gegenstand seiner tiefen Zuneigung gewesen. Qvigstad und Tromsø, das sind zwei Namen, die ebenso untrennbar miteinander verbunden sind wie - sagen wir - Immanuel Kant und Königsberg! Ausser der Zeit seiner Universitätsstudien hat sich Qvigstad im Lauf seines langen Lebens nur ein paar Jahre fern von seiner Heimatstadt aufgehalten. Diese Verbundenheit versteht wohl jeder beliebige, der auch nur als Turist nach Tromso gekommen und an einem sonnigen Sommertag die Strassen der am steilen Hang einer kleinen Insel erbauten Stadt bergauf gestiegen ist, bis er den Zauber der einzigartigen Aussicht geniessen konnte, die sich ron dort eröffnet. Aber ausserdem atmet Tromsø und die mit ihm verbundene Landschaft an der Küste Finnmarkens in hohem liass die bezaubernde Romantik der Geschichte: Diese Küste entlang, im Schutz einer nahen ununterbrochenen Inselreihe führte eine uralte Segelschiffahrtsstrasse nach dem Osten bis nach Bjarmaland, hier herrschte Ottar von Haalogaland über seine Güter und Renntierherden, an dieser düsteren Küste erschien das Lappenvolk aus der rätselhaften Dämmerung seiner Vorgeschichte, schloss Bekanntschaft nit den Skandinaviern und litt Bedrückung vonseiten der Tschuden, Quänen und Birkkerle. Hier gibt es für einen Chronisten genug zu erzählen! Qvigstad hat in allgemeinrerständlichen Aufsätzen Themen behandelt wie Ottar und andere bemerkenswerte historische Persönlichkeiten, den Ursprung der Stadt Tromsø, die Entwicklung der Nationalitätsverhältnisse jener Gegenden, die Geschichte der dortigen Landwirtschaft und des dortigen Handels sowie vor allem die der örtlichen Bildungs- und Schulverhältnisse. Noch 1952 verfasste der 99jährige Forscher den Aufsatz "Den norske befolkning i Finnmark og dens skoleforhold i eldre tid».

Die zwei Jahre, die Qvigstad ausser seiner Studienzeit fern ron Tromsø verbracht hat, wirkte er in Oslo als Kirchenund Erziehungsminister der von Wollert Konow gebildeten Regierung (1910-12). Dieser ehrenvolle Vertrauensposten wurde ihm als einer der hervorragendsten Persönlichkeiten des nordnorwegischen kulturellen Lebens übertragen. Eine so schroffe Abweichung ron Qrigstads gewohntem Lebensgang die Zeit seines Ministeramtes auch bedeutet haben muss, so ist aus ihr doch ein mit seinem spezialgebiet eng verbundenes wichtiges Ereignis zu vermerken: die Schaffung 
einer persönlichen Professur der finnisch-ugrischen Sprachen an der Universität Oslo für den Dozenten Konrad Nielsen. Dieser Vorschlag fand durchaus nicht einmütige Unterstützung; im Storthing bekämpfte ihn sogar ein Abgeordneter aus Nordnorwegen. Offensichtlich trug die sachliche Rede des Kultusministers entscheidend zur positiven Stellungnahme der Reichstagsmehrheit bei. "Es ist zwar so, dass im Jahr $1897^{1}$ die Professur für die lappische und finnische Sprache aufgehoben wurde. Aber ich glaube, dass nach 1897 die Meinungen in dieser Beziehung sich geändert haben. Jetzt sieht man, dass es in diesen Dingen noch viel zu erforschen gibt», äusserte er laut einem Zeitungsbericht unter anderem. Die allgemeine Einstellung hat sich nachher immer weiter in einer erfreulichen Richtung entwickelt, wie daraus hervorgeht, dass die betreffende persönliche Professur im Jahr 1946 in ein Ordinariat umgewandelt wurde.

Im Jahr 1910 war in dem ron Anders Larsen geleiteten Blatt "Sagai Muittalægje» folgender Absatz unter der ய̈berschrift "Eine gute Regel» veröffentlicht:

"Staatsrat Qvigstad, der ehemalige Leiter des Tromsøer Seminars, hatte die Gewohnheit, seine Schüler zu folgender Arbeitsweise aufzufordern: Das, was noch keine Eile hat, tu' ich zuerst, damit ich dann Zeit habe, wenn eine eilige Arbeit kommt. ${ }^{2}$

Wenn ein Mensch diese Regel befolgen würde, so würde er in seinem Leben viel schaffen. Qvigstad selbst hat wohl sicherlich diese Regel beachtet, denn er hat Zeit gefunden, viel zu lernen und neben seiner Schularbeit zu forschen und zu schreiben, so dass man seine Arbeitskraft bewundern muss.»

Staunen und Bewunderung erweckten also schon vor 43 Jahren Qvigstads Leistungen bei den Zeitgenossen. Noch mehr bewundern wir sie, die wir haben erleben dürfen, dass seine Arbeitskraft noch im Alter ron hundert Jahren andauert, die wir die Periode des grossen Anstiegs seiner Veröffentlichungstätigkeit gesehen haben. Als Qvigstad nach seiner Emeritierung endlich dazu $\mathrm{kam}$, sich auf seine Wissenschaft und auf die Bearbeitung seiner Sammlungen zu konzentrieren, war diese Arbeit nach menschlicher Voraussicht schon eilig, aber er führte sie in glänzender Weise zu Ende. Mit einem Gefühl tiefer Befriedigung kann der Jubilar nun seinen Lebensabend verbringen. Den hundertsten Meilenstein seiner langen Wanderung hat er in geistiger Frische

1 D. h. nach dem Tode von J. A. Friis.

2 Der Ausspruch hat in der Originalsprache (im Norwegischen) offenbar Gedichtform gehabt. 
und - mit Ausnahme seiner geschwächten Sehkraft - auch in recht gutem körperlichen Kräftezustand passiert.

Der bescheidene, stets freundliche und hilfsbereite Forscher hat im Verlauf seines Lebens viele Ehrungen erfahren. So ist er Ehrenmitglied der Finnischen Akademie der Wissenschaften, der Finnisch-ugrischen Gesellschaft und der Kalevalagesellschaft. In Norwegen hat $u$. a. das Tromsøer Huseum, auf dessen Kosten ein beträchtlicher Teil von Qvigstads Untersuchungen veröffentlicht worden ist, eine goldene Qvigstad-Medaille prägen lassen, die als hohe Auszeichnung für grosse Verdienste um die Erforschung der Lappen verliehen werden soll.

Loapas dat gīto ěuož̆̌̆o.

ERKKI ITKONEN.

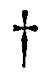

\section{Jalo Kalima.}

Gedenkrede, gehalten in der sitzung der Finnisch-ugrischen Gesellschaft am 20. 9. 1952.

Die Finnisch-ugrische Gesellschaft ist in der Zeit nach ihrer letzten Sitzung unerwartet von einem schweren Verlust betroffen worden. Das Mitglied des Vorstands der Gesellschaft, der Professor der slawischen Philologie Jalo Lahja Kalima starb an 30. Juni dieses Jahres. Sein Gesundheitszustand war schon im vergangenen Winter und Frühling schwankend, aber er selbst sowie seine Angehörigen und Freunde hofften, dass die Ruhe des Sommers wieder kräftigend auf ihn wirken würde. Diese Hoffnungen haben jedoch getrogen. Am 6. Juli wurde der Verstorbene in die Erde des Kirchhofs von Askola gebettet.

Jalo Kalima wählte offenbar schon in seiner Jugend die Laufbahn des Wissenschaftlers. Er studierte an der Universität u. a. slawische und finnisch-ugrische Sprachwissenschaft. Sicherlich unter Anleitung seiner Lehrer J. J. Mikkola, Heikki Paasonen und Yrjö Wichmann wählte er zum Thema seiner "Pro Gradu»-Schrift die Klärung der aus dem Russischen stammenden Wörter der syrjänischen Sprache. So kam er in einen Themenkreis, der sein ganzes Leben hindurch sein wichtigstes Tätigkeits- und Forschungsgebiet bleiben 